\title{
Influencia de factores edáficos en el crecimiento de una plantación de Pinus greggii Engelm. en Santiago de Anaya, Hidalgo, México
}

\section{Influence of edaphic factors on the growth of a plantation of Pinus greggii Engelm. in Santiago de Anaya, Hidalgo, Mexico}

\author{
Pedro Antonio Domínguez-Calleros', Rodrigo Rodríguez-Laguna², Juan Capulín-Grande², Ramón Razo-Zárate² y \\ Manuel Antonio Díaz-Vásquez ${ }^{*}$
}

1 Universidad Juárez del Estado de Durango. Facultad de Ciencias Forestales. Durango, Dgo., México.

\begin{abstract}
2 Universidad Autónoma del Estado de Hidalgo. Instituto de Ciencias Agropecuarias. Tulancingo, Hgo.,

* Autor de correspondencia. mdiaz@ujed.mx
\end{abstract} México.

\section{RESUMEN}

El objetivo de esta investigación fue evaluar la supervivencia y crecimiento de una plantación de Pinus greggii establecida en 2003 en un terreno (2.1 ha) con suelo degradado y escasa precipitación. En especial se evaluó el efecto de las características edafológicas del sitio en el crecimiento de la plantación. Se registraron también la cobertura, la regeneración natural y el material combustible. Para esto, se establecieron 9 sitios circulares de 1/10 ha, en tres bloques de acuerdo a la pendiente del terreno. La supervivencia promedio fue de $91 \%$, con mejores porcentajes en los bloques medio e inferior de la parcela. La altura, diámetro y volumen arrojaron diferencias significativas entre los sitios y bloques, los mayores valores e incrementos se registraron en los árboles de los sitios inferiores del terreno que muestran mayor fertilidad. En promedio la altura fue de $9.4 \mathrm{~m}$, el diámetro de $14.4 \mathrm{~cm}$ y el volumen $60.2 \mathrm{~m}^{3}$ r.t.a. ha ${ }^{-1}$ (metro cúbico de madera en rollo total del árbol en una superficie de $1 \mathrm{ha}$ ). En las partes altas del terreno se registró la menor cobertura vegetal (9.6\% del suelo), favoreciendo con esto la presencia de elementos vegetales propios de sitios con alta luminosidad, como los magueyes, registrándose en promedio 400 individuos por hectárea. Respecto al material combustible, solo se registró en los sitios inferiores y medios, siendo la biomasa combustible $\geq 5 \mathrm{~cm}$ de diámetro la de mayor cantidad (69.2 $\left.\mathrm{kg} \mathrm{ha}^{-1}\right)$. Dado que este material se encuentra disperso, no pone en riesgo de incendios a la plantación. Se recomienda realizar preaclareos y podas en los árboles que quedaran hasta el final del turno. Estas acciones reducirán la competencia y aumentaran la calidad de la madera, además de mejorar las condiciones edáficas del sitio mediante la incorporación del material vegetal al suelo.

PAlabras Clave: áreas degradadas, combustibles en bosques, fertilidad de suelos forestales, plantaciones forestales, reforestación, silvicultura en plantaciones.

\section{ABSTRACT}

The aim in this research was to evaluate survival and growth of a Pinus greggii plantation established since 2003, in a site (2.1 ha) with degraded soil and scarce precipitation. In particular, site soil characteristics and its influence on the development of the plantation were assessed. Vegetation coverage, natural regeneration and fuel load were also recorded. For this, nine circular sampling plots (1/10 ha) were measured in three random blocks according to the slope. Average survival was $91 \%$, with better percentages in the middle and lower blocks of the plantation. Height, diameter and volume showed significant differences among blocks and subsites; larger values and increments were

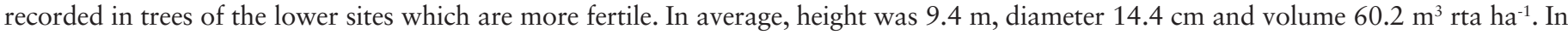
the upper portions of the land the lower vegetation cover was found (9.6 \% of the soil), favoring the natural regeneration of plants typical in sites with high luminosity, like agaves, registering an average of 400 individuals per hectare. With respect to fuel material, this was found only in the inferior and middle subsites, the most abundant being the material with more than $5 \mathrm{~cm}$ in diameter $\left(69.2 \mathrm{~kg}\right.$ ha $\left.{ }^{-1}\right)$. Since this fuel is dispersed all around, it does not represent a threat of fire to the plantation. It is recommended to perform pre thinning and pruning to reduce competition among trees and incorporate organic matter to the soil in order to improve its conditions. These actions will reduce competition and increase the wood quality, as well as improve the edaphic conditions of the site by incorporating the plant material into the soil.

KEYWORDS: degraded areas, forests fuels, forest soil fertility, forest plantations, reforestation, forestry plantations. 


\section{INTRODUCCIÓN}

En el Valle del Mezquital Hidalgo, México, las actividades antropogénicas efectuadas durante la década de los ochenta ocasionaron una fuerte reducción de la cobertura vegetal en los ecosistemas forestales y, con ello, una disminución en los servicios ambientales que los bosques ofrecen. Las plantaciones forestales establecidas por diversas dependencias federales como la Coordinación General del Plan Nacional de Zonas Deprimidas y Grupos Marginados (Coplamar), el Fideicomiso de Riesgo Compartido (Firco) y en años posteriores la Comisión Nacional Forestal (Conafor) cumplen más bien funciones sociales (Varela, 2006). Las actividades de investigación con propósitos de restauración ecológica del área se iniciaron desde 1993, las instituciones que han registrado resultados en este rubro son: la Universidad Autónoma del Estado de Hidalgo, el Colegio de Postgraduados y la Universidad Autónoma de Chapingo (Secretaría de Desarrollo Social - Comisión Nacional de Zonas Áridas, 1994). Estas investigaciones permitieron la creación del Centro Piloto de Conservación de Suelos y Desarrollo Rural en el Alto Mezquital, en 1996, con la participación de la Universidad Nacional Autónoma de México (UNAM). Entre los trabajos realizados destacan diferentes obras de conservación de suelos y agua, la reintroducción de especies de fauna y las reforestaciones con diversas especies vegetales (Varela, 2006). La especie utilizada con más frecuencia y éxito en las plantaciones forestales del área ha sido Pinus greggii Engelm. Investigaciones de Muñoz y Vargas (1988), Domínguez, Návar y Ortíz (2001), De los Ríos, Hoogh y Návar (2009), Gómez et al. (2012) y Lopez, Ramírez, Plascenciay Jasso (2004) señalan que este pino muestra una buena adaptación a condiciones adversas de clima y suelo, por lo que se recomienda para reforestaciones con propósitos de restauración de suelos. Sin embargo, nada se sabe de su comportamiento silvícola en plantaciones. Por esta razón, se realizó el presente trabajo en reforestaciones con esta especie en el parque recreativo El Porvenir, en Santiago de Anaya, Hidalgo. En esta plantación, Pinus greggii mostró buenos rendimientos en cuanto a supervivencia y crecimiento. Los resultados permiten primeramente, contar con un diagnóstico que señala la supervivencia del arbolado, así como conocer sus características dasométricas. Adicionalmente, proporcionan a los responsables del parque, elementos que apoyen la toma de decisiones en cuanto al manejo forestal de los sitios plantados, de manera que se propicie la regeneración natural, un mejor crecimiento del arbolado y un incremento en la superficie plantada, así como la supervivencia de las plantaciones con esta especie en la región.

\section{OBjetIVOS}

El objetivo principal de este trabajo fue evaluar la situación actual de una plantación con Pinus greggii Engelm. en Santiago de Anaya, Hidalgo. Por otra parte, se hace una evaluación de las condiciones edafológicas del sitio y el material combustible presente.

\section{MATERIALES Y MÉTODOS}

\section{Ubicación del área de estudio y los sitios}

El área de estudio se ubica en el paraje "La Manga Vieja", en el Parque Recreativo El Porvenir, al norte de la comunidad con el mismo nombre en Santiago de Anaya, Hidalgo, entre las coordenadas geográficas $20^{\circ} 25^{\prime} 18.4^{\prime \prime}$ y $20^{\circ} 30^{\prime} 2.4$ " de latitud Norte y $98^{\circ} 57^{\prime} 07^{\prime \prime}$ y $98^{\circ} 53^{\prime} 04^{\prime \prime}$ de longitud Oeste.

El clima es (BS1 kw $(\mathrm{w}))$ templado semiseco con verano cálido y lluvias en esta estación, según la clasificación de Köppen, modificada por García (1988), la temperatura media anual es de $17.23{ }^{\circ} \mathrm{C}$. La precipitación promedio anual es de $259.42 \mathrm{~mm}$ (Varela, 2006).

El tipo de suelo predominante es la Rendzina, caracterizado por su poca profundidad, de consistencia pegajosa y textura arcillosa, que se desarrolla sobre rocas calizas. Su susceptibilidad a la erosión es moderada, sobre todo en laderas pronunciadas (Varela, 2006).

Se establecieron nueve sitios circulares de $1000 \mathrm{~m}^{2}$, en tres bloques (tres por cada bloque), lo que representó 


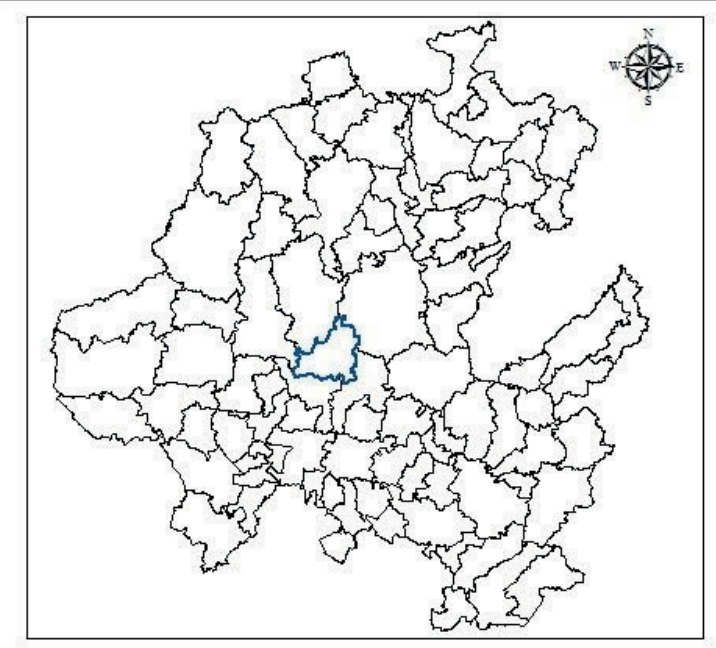

\section{Simbologia}

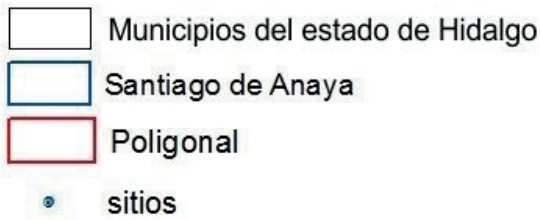

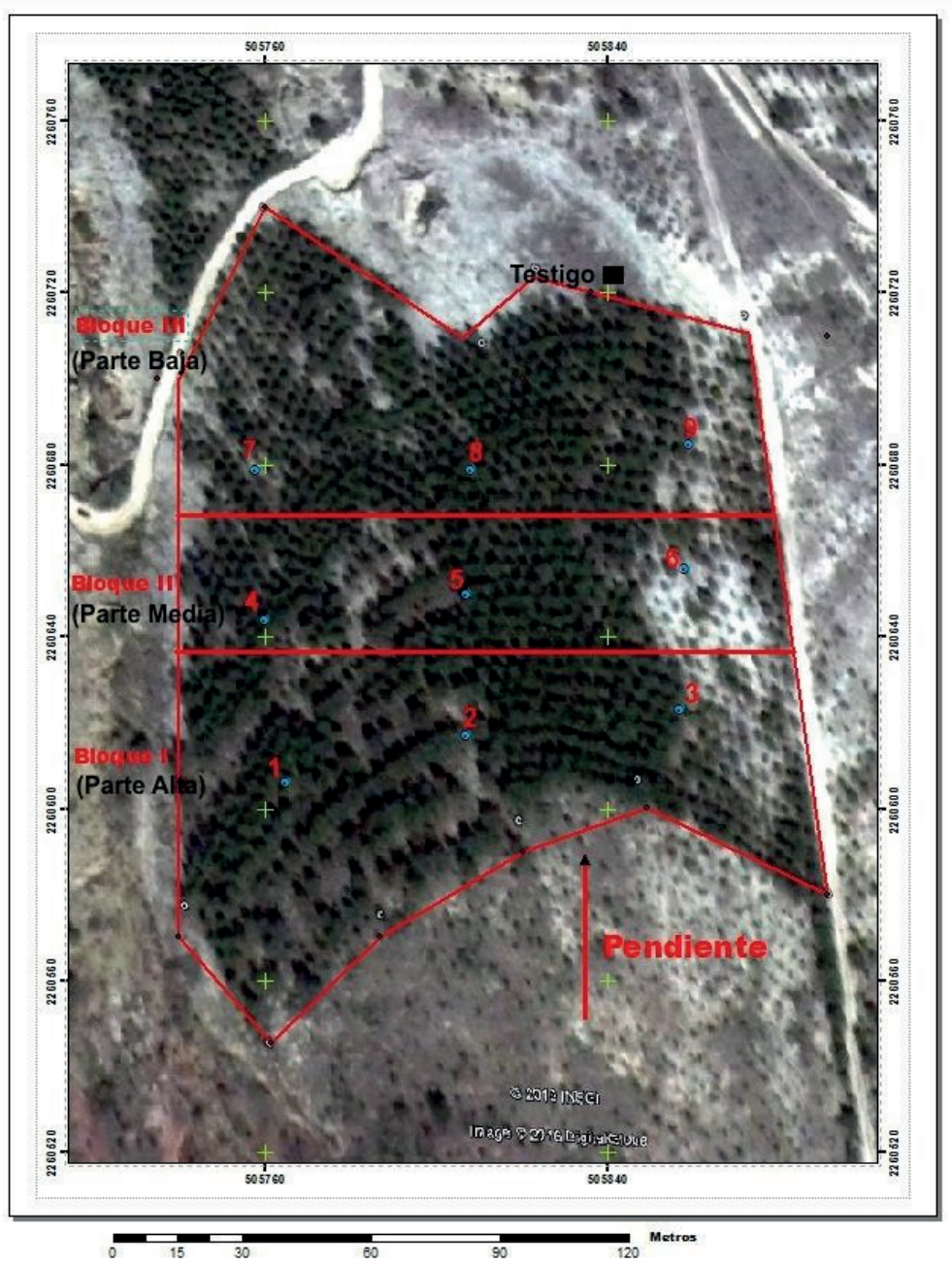

Figura 1. Área de estudio.

un muestreo de $42.5 \%$ de la superficie plantada originalmente (Fig. 1). En la elección de la forma y tamaño de los sitios se siguió la metodología propuesta por Murillo y Camacho (1997).

Caracterización del suelo. Para esta actividad se realizaron perfiles de suelo en las partes alta, media y baja del sitio dentro de la plantación y un testigo fuera de ella. Se tomaron muestras de suelo en cada bloque y testigo, a tres profundidades $(0 \mathrm{~cm}-5 \mathrm{~cm}, 6 \mathrm{~cm}-10 \mathrm{~cm}$ y $11 \mathrm{~cm}-15 \mathrm{~cm})$ con tres repeticiones. Por falta de recursos no fue posible tomar la humedad del suelo ni en los sitios ni en los bloques en diferentes etapas durante el año. A estas muestras de suelo se realizaron análisis físicos y químicos con el procedimiento descrito en la NOM-021-SEMARNAT-2000 (Secretaría de Medio Ambiente y Recursos Naturales [Semarnat], 2002).

Variables evaluadas. Para el registro de la supervivencia se tomó en cuenta el espaciamiento original de plantación que fue de $4 \mathrm{~m} \times 4 \mathrm{~m}$, lo que origina 225 árboles/ha. La altura $(\mathrm{m})$ fue tomada con un VERTEX® y el diámetro $(\mathrm{cm})$ con una cinta diamétrica. Para estimar el volumen se utilizó la formula señalada por Muñoz et al. (2012), la cual es: $\mathrm{V}=0.000137940$ (D) $)^{1.671395792}$ $(\mathrm{H})^{0.916603698}$, donde: $\mathrm{V}=$ Volumen $\left(\mathrm{m}^{3}\right), \mathrm{D}=$ Diámetro (cm) y H = Altura (m). La cobertura del suelo (\%) se calculó con una cuerda (17.84 m), la cual fue compensada 
para cada sitio según la pendiente de estos y marcada con siete nudos $(2.5 \mathrm{~m})$ y "tirada" en cuatro direcciones $(\mathrm{N}, \mathrm{S}, \mathrm{E}$ y $\mathrm{O})$ al azar. De tal manera que en cada sitio se tomaron en cuenta 28 puntos que tocaron la superficie del suelo. Se midió la profundidad de la hojarasca con un clavo graduado en centímetros. La regeneración natural en los sitios se registró en una superficie de $125 \mathrm{~m}^{2}$ dentro de cada sitio de muestreo; para esto se consideraron todas las especies vegetales presentes menores a $1.5 \mathrm{~m}$ de altura. La carga de combustible se contabilizó en una superficie de $250 \mathrm{~m}^{2}$ elegida al azar en cada sitio, separando material grueso $(\geq 5 \mathrm{~cm})$, delgado $(\leq 5 \mathrm{~cm})$ y conos encontrados en la superficie del suelo.

Los datos fueron manejados en Excel® y evaluados mediante análisis de varianza y comparación de medias, con el procedimiento GLM de SAS $®$

\section{RESULTADOS Y DISCUSIÓN}

\section{Caracterización edáfica del sitio}

Los resultados encontrados en la caracterización física y química del suelo de acuerdo con el gradiente de pendiente del sitio se muestran en las tablas 1 y 2 . Se observa que la pendiente tiene un papel muy importante en la acumulación nutrimental (Ramos, Castro y Sánchez, 2015); la parte alta presenta los menores valores con diferencia estadística de MO, C, P, K, Ca y Mg respecto a la parte baja; esto debido principalmente al escurrimiento del agua que arrastra partículas de suelo que llevan nutrimentos (Foth, 1990). Para el caso del N y del $\mathrm{pH}$ esa tendencia desaparece, ya que la parte alta, media, baja y el testigo presentan similitud en valores (Tabla 1).

TABla 1. Caracterización química del suelo en el área de estudio, Santiago de Anaya, Hidalgo.

\begin{tabular}{|c|c|c|c|c|c|c|c|c|}
\hline \multirow[t]{2}{*}{$\begin{array}{c}\text { Posición/profundidad } \\
\text { de capas (cm) }\end{array}$} & $p H$ & $M O$ & C & $N$ & $P$ & $K$ & $\mathrm{Ca}$ & $M g$ \\
\hline & & & $\%$ & & $m g \mathrm{~kg}^{-1}$ & \multicolumn{3}{|c|}{ Cmoles $\mathrm{kg}^{-1}$} \\
\hline \multicolumn{9}{|l|}{ Parte alta } \\
\hline $0-5$ & $7.1 \mathrm{a}$ & $2.7 \mathrm{~b}$ & $1.5 b$ & $0.20 a$ & 8.0ab & 0.53abcd & $46.6 \mathrm{bc}$ & $1.20 a b$ \\
\hline $6-10$ & $7.6 a$ & $0.7 b$ & $0.9 b$ & $0.13 a$ & $5.6 a b$ & O.40cd & $43.2 \mathrm{c}$ & $0.83 a b$ \\
\hline $11-15$ & $7.4 a$ & $0.8 b$ & $0.4 b$ & $0.10 a$ & $3.3 b$ & O.30d & 43.1c & $0.73 b$ \\
\hline \multicolumn{9}{|l|}{ Parte media } \\
\hline $0-5$ & 7.0a & $9.5 a b$ & 5.3ab & $0.22 a$ & $12.3 a$ & $0.83 a b$ & $53.6 a b$ & $1.70 a b$ \\
\hline $6-10$ & $7.6 a$ & $6.5 a b$ & 3.7ab & $0.19 a$ & $15.3 a$ & $0.5 a b c d$ & 47.4bc & $0.86 a b$ \\
\hline $11-15$ & 7.1a & 6.3ab & $3.6 a b$ & $0.18 a$ & $5.3 a b$ & $0.43 b c d$ & 49abc & 0.70b \\
\hline \multicolumn{9}{|l|}{ Parte baja } \\
\hline $0-5$ & 7.1a & $24.0 a$ & 13.9a & $0.19 a$ & 10.3ab & $0.90 a$ & $57.9 a$ & $1.93 a$ \\
\hline $6-10$ & $7.8 a$ & $22.5 a$ & $13.0 a$ & $0.15 a$ & $5.3 a b$ & $0.33 d$ & $43.9 b c$ & $0.66 b$ \\
\hline $11-15$ & $7.8 a$ & 13.6ab & 7.9ab & $0.14 a$ & 6.0ab & $0.36 \mathrm{~cd}$ & $44.3 b c$ & $0.63 b$ \\
\hline \multicolumn{9}{|l|}{ Testigo } \\
\hline $0-5$ & $7.9 a$ & $5.3 a b$ & $2.9 a b$ & $0.23 a$ & $9.6 a b$ & $0.73 a b c$ & 53.2abc & $1.13 a b$ \\
\hline $6-10$ & $7.7 a$ & $4.7 \mathrm{~b}$ & $2.4 \mathrm{~b}$ & $0.19 a$ & 8.3ab & $0.7 a b c$ & $53 a b c$ & $0.90 a b$ \\
\hline $11-15$ & $7.5 a$ & $4.4 \mathrm{~b}$ & $2.1 \mathrm{~b}$ & $0.16 a$ & $5.6 a b$ & $0.63 a b c$ & 49.2abc & $0.73 b$ \\
\hline
\end{tabular}


Los valores más contrastantes y con diferencia estadística se observan cuando el análisis es en función de la profundidad. Se detecta en todas las variables evaluadas, que en la capa superficial $(0 \mathrm{~cm}-5 \mathrm{~cm})$ presenta los mayores contenidos y a mayor profundidad $(11 \mathrm{~cm}-15$ $\mathrm{cm})$ estos decrecen. Esto se explica porque en la capa superficial tanto de los sitios como del testigo, se lleva a cabo la acumulación de los restos orgánicos y su posterior descomposición (Chavez, Merino, Vázquez y García, 2014), lo que propicia la liberación de nutrimentos que incrementan su contenido y disponibilidad para las plantas (Gallardo, Coverlo, Morillas y Delgado, 2009), logrando un mayor crecimiento. La NOM-021-SEMARNAT-2000 indica los criterios de clasificación de los parámetros del suelo, muestra que el $\mathrm{pH}$ no tiene diferencias según el gradiente y la profundidad y se clasifica de neutro a ligeramente alcalino; la $\mathrm{MO}$ y el $\mathrm{C}$ tienen un contenido en la parte alta de bajo a medio, en la media y alta contenido muy alto y en el testigo alto; el $\mathrm{N}$ tiene un contenido de medio a alto a lo largo del gradiente; el $\mathrm{P}$ presenta en todos los casos contenido de medio a alto en la capa superficial y a mayor profundidad contenidos de medio a bajo; el $\mathrm{K}$ en la parte alta muestra contenido medio, en partes media, baja y testigo de medio a alto; El contenido de $\mathrm{Ca}$ en todo el gradiente y profundidad muestra contenido alto; finalmente el $\mathrm{Mg}$ tiene contenido bajo en la parte alta y testigo, y de bajo a medio en la parte media y baja.

Las características físicas del suelo en el área de estudio muestran pocos cambios (Tabla 2). Se observaron diferencias en el contenido de partículas, sin llegar a modificar la clase textural, a excepción de la parte media en profundidad de $6 \mathrm{~cm}-10 \mathrm{~cm}$ que mostró mayor contenido de arcilla. La densidad aparente (Dap) fue menor

Tabla 2. Caracterización física del suelo en el área de estudio, Santiago de Anaya, Hidalgo.

\begin{tabular}{|c|c|c|c|c|c|c|c|}
\hline \multirow{2}{*}{$\begin{array}{c}\text { Posición/profundidad } \\
\text { de capas (cm) }\end{array}$} & \multirow{2}{*}{$\begin{array}{c}\text { Dap } \\
g \mathrm{~cm}^{-3}\end{array}$} & \multicolumn{3}{|c|}{ Proporción de partículas (\%) } & \multirow[t]{2}{*}{ Clase Textural } & \multicolumn{2}{|c|}{ Descripción del color } \\
\hline & & Arena & Limo & Arcilla & & Clave & Color \\
\hline \multicolumn{8}{|l|}{ Parte alta } \\
\hline $0-5$ & $0.98 a$ & 55 & 19 & 26 & F-A-A & 10YR5/2 & Café grisaceo \\
\hline $6-10$ & & 55 & 18 & 27 & F-A-A & 1OYR7/2 & Gris claro \\
\hline $11-15$ & & 54 & 18 & 28 & F-A-A & 10YR8/3 & Café pálido \\
\hline \multicolumn{8}{|l|}{ Parte media } \\
\hline $0-5$ & $0.90 a$ & 56 & 19 & 25 & F-A-A & 10YR6/3 & Café pálido \\
\hline $6-10$ & & 51 & 14 & 35 & $A-A$ & 10YR6/2 & Gris parduzco \\
\hline $11-15$ & & 53 & 18 & 29 & F-A-A & 10YR7/2 & Gris claro \\
\hline \multicolumn{8}{|l|}{ Parte baja } \\
\hline $0-5$ & $0.88 a$ & 58 & 15 & 27 & F-A-A & 10YR6/2 & Gris parduzco \\
\hline $6-10$ & & 53 & 21 & 26 & F-A-A & 10YR6/2 & Gris parduzco \\
\hline $11-15$ & & 53 & 21 & 26 & F-A-A & 1OYR7/2 & Gris claro \\
\hline \multicolumn{8}{|l|}{ Testigo } \\
\hline $0-5$ & $0.98 a$ & 61 & 17 & 22 & F-A-A & 10YR4/3 & Café \\
\hline $6-10$ & & 58 & 15 & 27 & $F-A-A$ & 10YR4/2 & Café grisáceo \\
\hline $11-15$ & & 56 & 11 & 33 & F-A-A & 10YR5/2 & Café grisáceo \\
\hline
\end{tabular}

F-A-A = franco arcillo arenoso; A-A = Arcilla arenosa; Dap= densidad aparente. 
en las partes media y baja de la plantación, aunque la prueba de Tukey $(\mathrm{P} \leq 0.05)$ no indica diferencias estadísticas en los valores promedio. Esto se explica por la acumulación de materia orgánica que propicia un aumento de volumen en el suelo y una microporosidad (Mora y Lázaro, 2014). El color va de gris claro hasta café sin cambios bruscos. Esta ligera variación se debe a que los cambios físicos y químicos en el suelo son muy lentos por la baja meteorización de la roca, aunado a que en el área de estudio existe poca precipitación (Brady y Weil, 2016; Chaplot, Vliet, Walter, Curmi y Cooper, 2003; Muñoz Iniestra, Chávez Mosqueda, Godínez Álvarez y Cuéllar Arellano, 2017).

\section{Supervivencia}

Debido al número de árboles en los sitios no fue posible realizar comparaciones estadísticas, por lo que este parámetro solamente se refiere en valores porcentuales. Los valores de supervivencia de los sitios ubicados en la parte inferior de la parcela fueron mayores, $100 \%$ y $85.7 \%$ en el bloque 3 y 2 respectivamente, que los ubicados en los sitios de la parte alta, $85 \%$ en el bloque 1. En la parte inferior del terreno, la escorrentía es mayor ya que en forma natural el agua fluye hacia las partes bajas, reflejado esto en mayor supervivencia y crecimiento de las plantas (Lasanta y Ortigosa, 1984; Foth 1990). Las investigaciones realizadas por Rodríguez, Meza, Vargas y Jiménez (2009) y López et al. (2004) no señalan las condiciones de la calidad de la planta, técnicas de plantación y procedencias utilizadas, a pesar de que estos factores influyen positivamente en el rendimiento de la plantación (Domínguez et al., 2001). Por lo anterior, no es posible realizar una comparación de los resultados de los autores antes mencionados con los de esta investigación. En El Porvenir, se utilizaron arbolitos cultivados durante 12 meses en bolsas de polietileno de $400 \mathrm{~cm}^{3}$ llenadas con tierra de monte y se establecieron en cepas elaboradas seis meses antes de la época de plantación (Varela, 2016). Después de 13 años, el porcentaje de supervivencia puede considerarse excelente $(91 \%)$ para todo el rodal.

\section{Altura}

El análisis de varianza para este parámetro arrojó diferencias significativas entre los sitios ( $\mathrm{P} \leq 0.0001)$. En la figura 2 se muestran la altura promedio de los árboles por sitio y sus desviaciones estándar. La prueba de Duncan formó tres grupos, en el primero se encuentran los árboles del sitio 6, sus promedios de altura fueron los mayores. En el segundo grupo se encuentran los sitios 4, 5, 7, 8 y 9, destacando entre estos, los árboles del sitio 7 ya que ocuparon el segundo lugar y su desviación estándar fue la más estrecha; en este grupo los árboles del sitio 5 ocuparon la tercera posición con la desviación estándar más amplia. La altura de los árboles de los sitios superiores (1, 2 y 3$)$ formó el tercer grupo, su altura promedio fue la más baja, coincidiendo con menor contenido de nutrimentos en el terreno y sus desviaciones estándar fueron similares.

El análisis de varianza entre los bloques arrojó diferencias significativas $(\mathrm{P} \leq 0.0001)$, siendo mayores en la parte media y baja de la pendiente. La altura promedio de toda la plantación fue de $9.4 \mathrm{~m}$. Aunque los valores registrados en este trabajo son muy distintos a los señalados por Domínguez Calleros et al. (2001); López et al. (2004); Aguilar (2009); Muñoz et al. (2011) y López Santiago (2013), puede presumirse una excelente adaptabilidad de Pinus greggii a sitios de baja fertilidad como los del área de estudio.

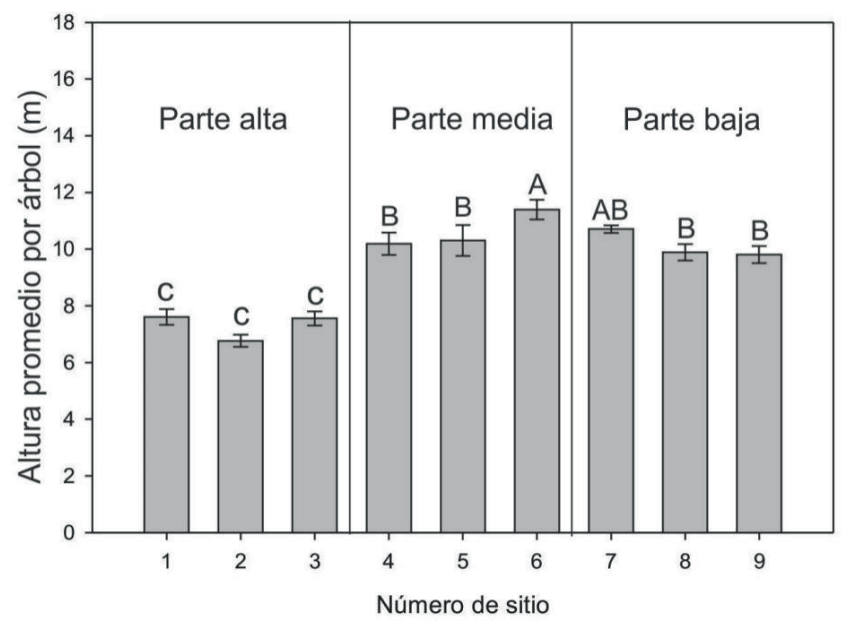

Figura 2. Altura y desviación estándar de los árboles en los sitios. 


\section{Diámetro}

En la evaluación de este parámetro, el análisis de varianza indica que existen diferencias significativas entre los sitios $(\mathrm{P} \leq 0.0001)$. El promedio y sus errores estándar se muestran en la figura 3. El diámetro promedio de toda la plantación es de $14.4 \mathrm{~cm}$. La prueba de Duncan forma 4 grupos, destacando los arboles del sitio 8 con el diámetro mayor y los del sitio 2 con el diámetro menor.

Existen diferencias significativas del diámetro entre los bloques ( $\mathrm{P} \leq 0.0001)$, teniendo los mayores valores los bloques ubicados en la parte media y baja del terreno.

Las diferentes procedencias de P. greggii, la calidad de planta, las técnicas de plantación, las condiciones edáficas y climáticas de los sitios plantados con esta especie señaladas en las investigaciones de López et al. (2004), Aguilar (2009) y Muñoz et al., (2011) impiden comparar los resultados con los observados en este trabajo. Sin embargo, los diámetros registrados reflejan la excelente adaptabilidad de la especie a sitios degradados.

En las plantaciones de El Porvenir se recomienda la aplicación de preaclareos y podas a fin de reducir la competencia por nutrientes y con esto la aparición de plagas forestales, como aconteció en una plantación de P. greggii en el Ejido Fontezuelas, municipio de Meztitlan, Hgo. (Varela, 2016).

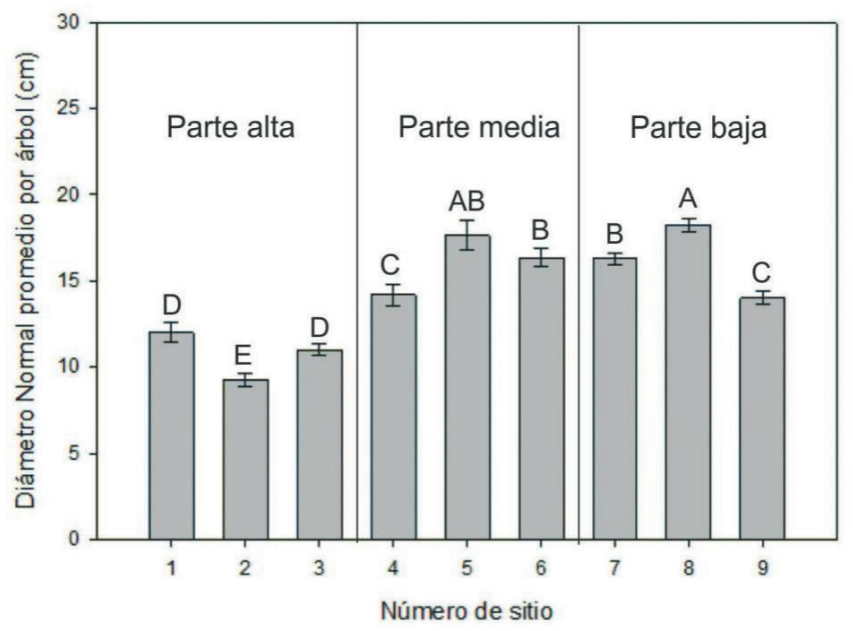

Figura 3. Diámetro y desviación estándar de los árboles en los sitios

\section{Volumen}

A los 13 años de establecida la plantación, el volumen promedio registrado fue de $60.2 \mathrm{~m}^{3}$ r.t.a. ha ${ }^{-1}$. El análisis de varianza señala que existen diferencias significativas entre los sitios ( $\mathrm{P} \leq 0.0001)$. La figura 4 muestra los valores promedio y las desviaciones estándar de este parámetro. En la prueba de Duncan se destacan los valores registrados en los sitios 5 y 2 con los volúmenes máximo y mínimo respectivamente. El análisis de varianza para este parámetro también detecta diferencias significativas $(\mathrm{P} \leq 0.0001)$ entre los bloques, registrándose los mayores valores en las partes media y baja de la pendiente. El volumen fue menor en los árboles de los sitios superiores. Sin embargo, dadas las condiciones del sitio en El Porvenir, el volumen registrado se considera aceptable.

En la tabla 3 se muestra cómo la producción de hojarasca fue menor en los sitos ubicados en la parte alta del terreno (1, 2 y 3); también aquí la profundidad promedio de la capa fue la más baja; por el contrario, se observó un mayor porcentaje de suelo desnudo o roca que en los demás sitios.

En la misma tabla 3 se presenta la regeneración natural registrada. Excepto en el sitio 2, se observaron elementos vegetales (magueyes y enebros) propios de sitios con intensa luminosidad y suelos delgados o someros, producto de su lenta intemperización o formación

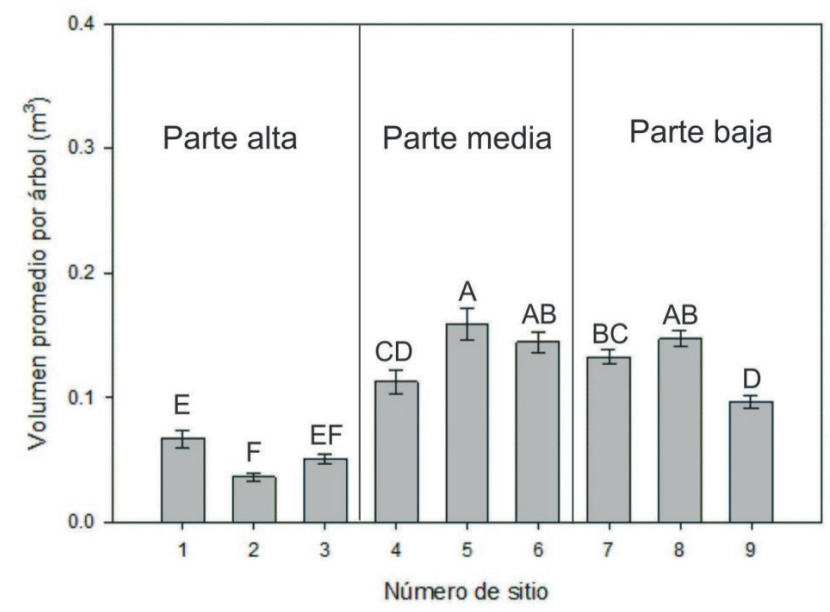

Figura 4. Volumen y desviación estándar de los árboles en los sitios. 
TAвla 3. Cobertura del suelo (\%), regeneración natural y material combustible en los sitios.

\begin{tabular}{|c|c|c|c|c|c|c|c|c|c|c|}
\hline \multirow[t]{3}{*}{ Ubicación } & \multirow[t]{3}{*}{ Sitios } & \multicolumn{3}{|c|}{ Cobertura } & \multicolumn{3}{|c|}{ Regeneración natural ( $N$ ha $\left.^{-1}\right)$} & \multicolumn{3}{|c|}{ Material combustible $\left(\mathrm{kg} \mathrm{ha}^{-1}\right)$} \\
\hline & & \multicolumn{2}{|c|}{ Hojarasca } & \multirow{2}{*}{$\frac{\text { Suelo-roca }}{(\%)}$} & \multirow[b]{2}{*}{ Pino } & \multirow[b]{2}{*}{ enebro } & \multirow[b]{2}{*}{ maguey } & \multirow[b]{2}{*}{$\geq 5 \mathrm{~cm}$} & \multirow[b]{2}{*}{$\leq 5 \mathrm{~cm}$} & \multirow[b]{2}{*}{ Conos } \\
\hline & & $(\%)$ & $(\mathrm{cm})$ & & & & & & & \\
\hline \multirow[t]{4}{*}{ Alta } & 1 & 10.8 & 2.3 & 89.2 & & & 400 & & & \\
\hline & 2 & 14.3 & 1.7 & 85.7 & & & & & & \\
\hline & 3 & 3.6 & 4.0 & 96.4 & & & 800 & & & \\
\hline & $\dot{x}$ & 9.6 & 2.7 & 90.4 & --- & --- & 400 & --- & --- & --- \\
\hline \multirow[t]{4}{*}{ Media } & 4 & 78.6 & 5.9 & 21.4 & 880 & & 80 & 28 & 10 & 48 \\
\hline & 5 & 71.4 & 3.5 & 28.6 & & & 800 & & & \\
\hline & 6 & 89.3 & 2.3 & 10.7 & 240 & 80 & 160 & 56 & 26 & 6 \\
\hline & $\dot{x}$ & 79.8 & 3.9 & 20.2 & 373 & 27 & 347 & 28 & 12 & 18 \\
\hline \multirow[t]{4}{*}{ Baja } & 7 & 75.0 & 4.2 & 25.0 & 80 & 640 & & 54 & 28 & 52 \\
\hline & 8 & 89.3 & 4.9 & 10.7 & 160 & 160 & 160 & 132 & 76 & 64 \\
\hline & 9 & 50.0 & 5.8 & 50.0 & 240 & & 240 & 76 & 32 & 6 \\
\hline & $\dot{x}$ & 71.4 & 4.9 & 28.6 & 160 & 267 & 133 & 87 & 45 & 41 \\
\hline
\end{tabular}

reciente (Porta, López y Roquero, 2008). De acuerdo con los parámetros evaluados de la tabla 1 , el contenido nutrimental de la parte alta y testigo es de bajo a medio; mientras que en la parte media y baja el contenido es de medio a alto (NOM-021-SEMARNAT-2000; Semarnat, 2002).

Aunque también en los sitios intermedios y bajos se observaron pinos, enebros y magueyes, estos fueron menores a $50 \mathrm{~cm}$ de alto, individuos jóvenes cuya permanencia en el bosque presumiblemente se verá afectada por las condiciones edáficas y silvícolas, sobre todo por el grosor o profundidad de la hojarasca de los sitios. La presunción anterior se basa en estudios realizados por Eckelmann (1990) y Domínguez (2000), quienes señalan que las raíces de las plántulas permanecen en la hojarasca y al no alcanzar mayores profundidades en el suelo para abastecerse de agua, la planta se seca una vez que la hojarasca pierde humedad.

Carga de combustibles. El conocimiento de los combustibles que existen en el bosque es una necesidad imperiosa para los encargados de la protección contra incendios forestales (Jardel P. et al., 2010), sobre todo aceptando que estos son un fenómeno ampliamente extendido en los ecosistemas terrestres del mundo. Los combustibles son cualquier sustancia o compuesto susceptible de encenderse y mantener un proceso de combustión (Morfín, Jardel, Alvarado y Michel, 2012), estos investigadores proponen diferentes métodos de muestreo según la estructura del bosque. Por tratarse de un rodal joven y de pequeña extensión, en El Porvenir se registró únicamente el material depositado en la superficie del suelo, los resultados se muestran en la tabla 3. Solo en la parte media e inferior de la parcela se registró material leñoso, la mayoría de él $\geq 5 \mathrm{~cm}$; hubo mayor cantidad en la parte inferior. Morfín et al. (2012) indican que estos restos vegetales intervienen en los incendios superficiales, subterráneos y en la combustión residual y son los que convencionalmente se han utilizado para cuantificar el comportamiento del fuego. Las cantidades registradas de material combustible en El Porvenir no ponen en riesgo de incendios al rodal. Sin embargo, se recomienda el manejo del material leñoso para evitar la incidencia 
del fuego en los próximos años y acelerar la descomposición de este material para la incorporación de nutrientes al sitio.

\section{CONCLUSIONES}

Los resultados de esta investigación indicaron mejor supervivencia y crecimiento de P. greggii en los sitios situados en la parte baja del terreno con mayor contenido nutrimental. La menor cobertura vegetal de los sitios de las partes altas permitió la presencia de elementos vegetales propios de suelos con condiciones difíciles. La mayor cantidad de material combustible, se registró en los sitios inferiores, aunque esta no pone en riesgo a la plantación por probables incendios. Se recomienda realizar preaclareos y podas y triturar el material para su integración al ciclo de nutrientes o la aplicación de fertilizantes al suelo para así mejorar el crecimiento de los árboles que se dejarán hasta el final del turno.

\section{RECONOCIMIENTOS}

Este trabajo fue posible gracias a la beca No. 265473 del CONACYT durante el segundo periodo de 2015 para la realización de una estancia sabática en el ICAP-UAEH que recibió uno de los autores. Se agradecen los comentarios del editor y revisores anónimos que fortalecieron el documento.

\section{REFERENCIAS}

Aguilar, C. J. (2009). Captura de carbono en una plantación de Pinus greggii Engelm., en Arteaga Coahuila. Tesis de Licenciatura no publicada, Universidad Autónoma Agraria Antonio Narro, Buena Vista, Saltillo, Coahuila, México. 60 p.

Brady, N. C. y Weil R. R. (2016). The nature and properties of soils (15a ed.). Columbus, EUA: Pearson Education, Inc.

Chaplot, V., Van Vliet-Lanoë, B., Walter, C., Curmi, P. y Cooper, M. (2003). Soil spatial distribution in the armorican massif, western France: Effect of soil-forming factors. Soil Science, 168, 856-868

Chávez V., B., Merino, A., Vázquez M., G. y García O. F. (2014). Organic matter dynamics and microbial activity during decomposition of forest floor under two native neotropi- cal oak species in a temperate deciduous forest in Mexico. Geoderma, 235-236, 133-145. doi: 10.1016/j.geoderma.2014.07.005

De los Ríos C., E., de Hoogh, R., and Navar Ch., J. J. (2009). Projections of carbon stocks in sites reforested with pinyon pine species in northeastern Mexico. Arid Land Research and Management, 23(4), 342-358. doi: $10.1080 / 15324980903231884$

Domínguez C., P. A. (2000). Fundamentos de Silvicultura. Linares N. L.: Universidad Autónoma de Nuevo León, Facultad de Ciencias Forestales.

Domínguez C., P. A., Návar Ch., J. J. y Loera O., J. A. (2001). Comparación del rendimiento de pinos en la reforestación de sitios marginales en Nuevo León. Madera y Bosques, 7(1), 27-35. doi: 10.21829/myb.2001.711316

Eckelmann, C. M. (1990). Untersuchungen zur naturverjüngung in natürlichen Kiefern-Eichen Wäldern der Sierra Madre Oriental im nordosten Mexikos. Unveröffentlichte Dissertation. Universität Göttingen. Alemania.

Foth, H. D. (1990). Fundamentals of Soils Science (8a ed.). EUA: John Wiley \& Sons.

Gallardo, A., Covelo, F., Morillas, L. y Delgado, M. (2009). Ciclos de nutrientes y procesos edáficos en los ecosistemas terrestres: especificidades del caso mediterráneo y sus aplicaciones para las relaciones suelo-planta. Ecosistemas, 18(2), 4-19.

García, E. (1988). Modificaciones al sistema de clasificación climática de Köppen (para adaptarlo a las condiciones de la República Mexicana). (3 ed.). México, D. F. UNAM, Instituto de Geografía.

Gómez R., M., Soto C., J. C., Blanco G., J. A., Sáenz R., C., Villegas, J. y Lindig C., R. (2012). Estudio de especies de pino para restauración de sitios degradados. Agrociencia, 46(8), 795-807.

Jardel P., E. J., Frausto L., J. M., Pérez S., D., Alvarado, E., Morfín R., J. E., Landa P., R., y Llamas C., P. (2010). Prioridades de investigación en manejo del fuego en México. México D.F.: Fondo Mexicano para la Conservación de la Naturaleza.

Lasanta M., T., y Ortigosa I., L. M. (1984). El papel de la escorrentía en la organización textural de suelos cultivados en 
pendiente: modelos en viñedos de La Rioja. Cuadernos de Investigación Geográfica, 10: 99-112. doi: 10.18172/ cig. 928 .

López S., D. (2013). Análisis del tamaño de muestra para evaluar biomasa aérea en Pinus greggii Engelm., en Arteaga, Coabuila, México. Tesis de licenciatura no publicada. Universidad Autónoma Agraria Antonio Narro. Buenavista, Saltillo, Coahuila, México.

López U., J., Ramírez H., C., Plascencia E., O. y Jasso M., J. (2004). Variación en crecimiento de diferentes poblaciones de las dos variedades de Pinus greggii. Agrociencia, 38(4), 457-464.

Mora, J. L. y Lázaro, R. (2014). Seasonal changes in bulk density under semiarid patchy vegetation: the soil beats. Geoderma, 235-236: 30-38. doi: 10.1016/j. geoderma.2014.06.022

Morfin R., J. E., Jardel P., E. J., Alvarado C., E. y Michel F., J. M. (2012). Caracterización y cuantificación de combustibles forestales. Comisión Nacional Forestal-Universidad de Guadalajara. Guadalajara, Jalisco, México.

Muñoz F., H. J., Orozco G., G., Coria A., V. M., García S., J. J., Muñoz V., Y. Y., y Salvador C., G. (2011). Evaluación de Pinus pseudostrobus Lindl. y Pinus greggii Engelm. con dos densidades de plantación en Michoacán, México. Foresta Veracruzana, 13(1), 29-35.

Muñoz F., H. J., Velarde R., J. C., García M., J. J., Sáenz R., J. T., Olvera D., E. H., y Hernández R., J. (2012). Predicción de volúmenes de fuste total para plantaciones de Pinus greggii Engelm. Revista Mexicana de Ciencias Forestales, 3(14), 11-22.

Muñoz I., D. J., Chávez M., M., Godínez Á., H. O., y Cuéllar A., N. A. (2017). Cambios edáficos en islas de fertilidad y su importancia en el funcionamiento de un ecosistema del valle de Tehuacán Puebla, México. Revista Terra Latinoamericana, 35(2), 123-134.

Muñoz O., A. y Vargas H., J. J. (1988). Resistencia a sequía: II. Crecimiento y supervivencia en plántulas de cuatro especies de Pinus. Agrociencia, 72, 197-208.

Murillo, O. y Camacho, P. (1997). Metodología para la evaluación de la calidad de plantaciones forestales recién establecidas. Agronomía Costarricense, 21(2), 189-206.
Porta, J., López A., M. y Roquero de L., C. (2008). Edafología para la agricultura y el medio ambiente, ( $3^{\mathrm{a}}$ ed.). Madrid, España: Mundi-Prensa.

Ramos, D., Castro, V. y Sánchez, E. (2015). Caracterización de la vegetación a lo largo de una gradiente altitudinal en la comunidad de Cochahuayco, cuenca media del río Lurín, Lima. Revista Ecología Aplicada, 14(1), 11-25. doi:10.21704/rea.v14i1-2.78

Rodríguez L., R., Meza R., J., Vargas H., J. J., y Jiménez P., J. (2009). Variación en la cobertura de suelo en un ensayo de procedencias de Pinus greggii Engelm. en el cerro El Potosí, Galeana, Nuevo León. Madera y Bosques, 15(1), 47-59. doi: 10.21829/myb.2009.1511196

Secretaría de Desarrollo Social- Comisión Nacional de Zonas Áridas (1994). Plan de Acción para combatir la desertificación en México (PACD-MEXICO). México.

Secretaría de Medio Ambiente y Recursos Naturales [Semarnat] (2002). Norma Oficial Mexicana (NOM-021-SEMARNAT-2000) que establece las especificaciones de fertilidad, salinidad y clasificación de suelos, estudio, muestreo y análisis. México, D. F.: Diario Oficial de la Federación, segunda sección.

Varela P., A. (2006). Participación social en la restauración de áreas degradadas. Universidad Autónoma Chapingo. 33 p.

Varela P., A. (2016). El manejo integral de la microcuenca El Porvenir, Santiago de Anaya, Hidalgo. (Datos no publicados). Delegación Federal de la Semarnat del Estado de Hidalgo.

Manuscrito recibido el 12 de febrero de 2017. Aceptado el 12 de julio de 2017.

Este documento se debe citar como:

Domínguez-Calleros, P. A., Rodríguez-Laguna, R., Capulín-Grande, J., Razo-Zárate R. y Díaz-Vásquez, M. A. (2017). Influencia de factores edáficos en el crecimiento de una plantación de Pinus greggii Engelm. en Santiago de Anaya, Hidalgo, México. Madera y Bosques, 23(2), 145-154. doi: 10.21829/myb.2017.2321522 